\title{
Infectious Complications Following Solid Organ Transplantation
}

\author{
Alexis Guenette, DO ${ }^{a}$, Shahid Husain, MD, MS, FECMM, FRCP ${ }^{\mathrm{b}, *}$
}

\section{KEYWORDS}

- Infections $\bullet$ Solid organ transplant $\bullet$ Critical care $\bullet$ Intensive care unit (ICU) • Sepsis

\section{KEY POINTS}

- Solid organ transplant recipients are a complex group of patients who require a low threshold of suspicion regarding infection, and given their underlying immunosuppression, mortality and morbidity are high.

- Individually they require assessment of their net state of immunosuppression and subsequent thorough evaluation of infectious causes.

- Early diagnosis is key along with appropriate, tailored treatment.

\section{INTRODUCTION}

Immunosuppression plays an integral role in solid organ transplant (SOT) recipients because it increases graft survival; however, there are unintended consequences, such as infectious complications. One strategy aimed at assessing the functionality of the immune system consists of non-pathogen-specific immune monitoring, consisting of serum immunoglobulins, serum complement factors, peripheral blood lymphocyte subpopulations, soluble CD30, and iATP in CD4 ${ }^{+}$T cells. $^{1}$ Ideally, these would help to demonstrate one aspect of the overall "net state of immunosuppression." The "net state of immunosuppression" comprises all factors that may contribute to the risk of infection; this includes preexisting immune deficits, colonization with antimicrobial-resistant pathogens, immunosuppressive agents, acquired immunodeficiency, prior antimicrobial therapies, mucocutaneous barrier integrity, fluid collections, neutropenia, lymphopenia, and viral coinfections. ${ }^{2}$

Disclosure Statement: A. Guenette has no relationships with a commercial company that has a direct financial interest in subject matter or materials discussed in article or with a company making a competing product. S. Husain has received grants from Astellas, Pfizer, and Merck.

a Division of Infectious Disease, University Health Network, University of Toronto, 585 University Avenue, 11 PMB 138, Toronto, Ontario M5G 2N2, Canada; ${ }^{b}$ Division of Infectious Disease, Multi-Organ Transplant Program, University Health Network, University of Toronto, 585 University Avenue, 11 PMB 138, Toronto, Ontario M5G 2N2, Canada

* Corresponding author.

E-mail address: Shahid.Husain@uhn.ca 
Vaccinations, surgical prophylaxis, universal prophylaxis, preemptive or presymptomatic therapy, targeted prophylaxis, and education avoidance are preventative strategies that have been implemented in SOT recipients. Trimethoprim/sulfamethoxazole prophylaxis is given in most institutions for 3 months to a lifetime to prevent Pneumocystis pneumonia along with Toxoplasma gondii, Cyclospora cayetanensis, and many Nocardia and Listeria species. Antiviral prophylaxis along with nucleic acid-based assays to prevent cytomegalovirus (CMV) and other herpesvirus infections has also transformed posttransplant care. ${ }^{2}$

Infections in SOT recipients reflect the net balance between the recipient's epidemiologic exposures and immunosuppression. ${ }^{2}$ Alterations to the balance can be seen with antimicrobial prophylaxis, immunosuppression, and improved graft survival. ${ }^{2}$ This balance is also affected during a period of graft rejection or intensification of immunosuppression (Fig. 1). ${ }^{2}$ In this article, the authors review infectious syndromes encountered in intensive care units among SOT recipients.

\section{BLOODSTREAM INFECTIONS/SEPSIS}

Bloodstream infections (BSIs) are associated with poor outcomes along with being the leading cause of mortality and morbidity in SOT. ${ }^{3-5}$ Mortality as high as $24 \%{ }^{4,6,7}$ has been described, and in fact, once septic shock develops, mortality can reach $50 \%, 4,8$ although Kalil and colleagues ${ }^{9}$ demonstrated that there may be a decrease in mortality of transplant patients compared with nontransplant patients. It is thought that SOT recipients do not necessarily clinically behave in the same manner due to underlying immunosuppression, and in fact, tend to present with organ failure and thrombocytopenia during sepsis. ${ }^{10}$ Universal risk factors for sepsis, regardless of transplanted organ, are CMV serology mismatch, particularly positive donor to negative recipient, CMV disease, which inherently demonstrates an immunomodulatory effect predisposing recipients to higher rates of bacterial and fungal sepsis, prolonged duration of graft cold ischemia, prolonged duration of surgical transplantation procedure, and requirement of large amounts of blood transfusion. ${ }^{10,11}$ Management should consist of rapid initiation of intravenous antibiotics, rapid diagnosis, source control, aggressive search

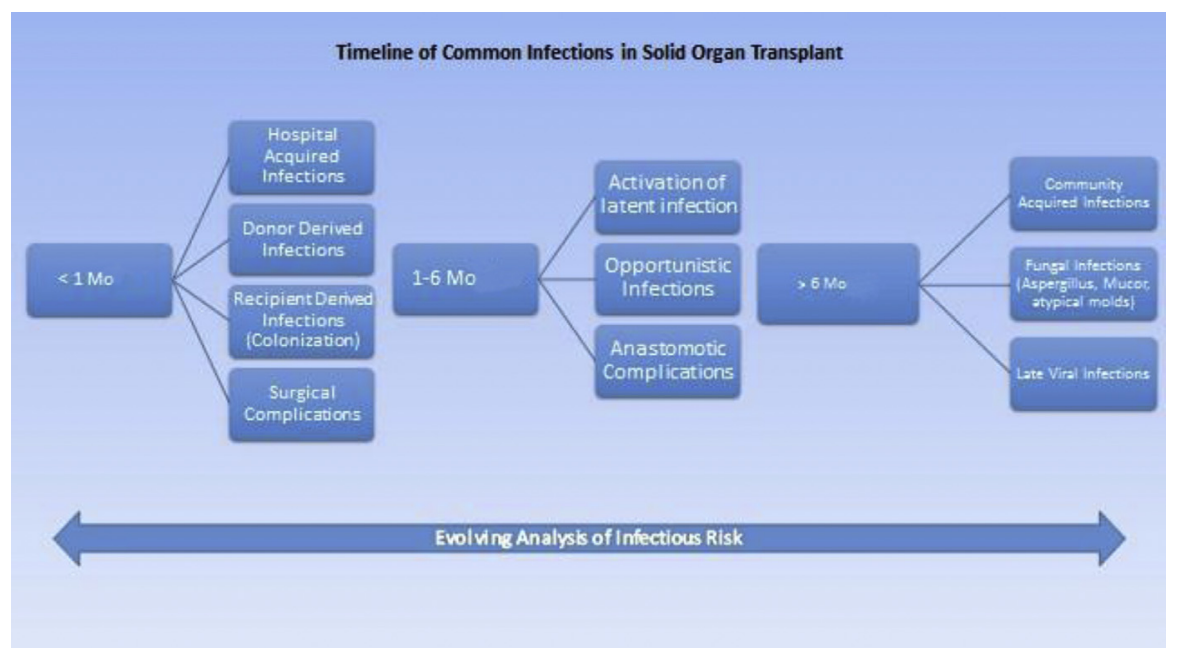

Fig. 1. Timeline of common infections in SOT. (Adapted from Fishman JA. From the classic concepts to modern practice. Clin Microbiol Infect 2014;20(suppl 7):4-9; with permission.) 
for pathologic condition that mimics severe sepsis, and reduction in immunosuppressive drugs. ${ }^{10}$

Nosocomial BSIs are associated with an even increased risk of septic shock and failure of cure when compared with other BSIs in SOT patients. ${ }^{3-5,8,12}$ Grampositive bacteria are the most frequent source of BSIs and are likely to be associated with intravascular catheters, especially in the nosocomial setting. ${ }^{3,4}$ However, with kidney transplant recipients (KTRs), gram-negative bacteria likely related to urinary tract infections (UTIs) are the primary source, regardless of the time period.

The overall incidence of multidrug-resistant organisms (MDRO) is increasing. MDRO gram-negative organisms accounted for about $14 \%$ of isolates. ${ }^{4}$ Fluconazoleresistant Candida spp accounted for up to $46 \%$ of cases of candidemia according to Moreno and colleagues. ${ }^{4,13}$ Vancomycin-resistant enterococci (VRE) have become an emerging pathogen with studies documenting an incidence of up to $20.5 \%$ of nosocomial Enterococcal spp BSIs consistent with VRE. These findings along with previous microbiological history and local antibiotic resistance patterns should be considered when determining empiric antimicrobial therapy.

Overall, there are limited studies on infective endocarditis (IE) in sold organ transplant recipients. The incidence of IE in a single center was $1 \%$, with an estimated 171-fold higher incidence as compared with the general population, ${ }^{14}$ with an overall mortality up to $57 \% .{ }^{15}$ There are limited data on the mode of infection and predisposing factors in SOT recipients. Underlying structural abnormalities may not appear to be a risk factor for IE as compared with the general population. ${ }^{14,15}$ According to Paterson and colleagues, ${ }^{15} 50 \%$ of infections were due to Aspergillus fumigatus or Staphylococcus aureus, and $4 \%$ were due to viridans streptococci, which is in contrast to the general population. A combination of antibiotic therapy as described in Infectious Diseases Society of America (IDSA) Guidelines, Infective Endocarditis in Adults: Diagnosis, Antimicrobial Therapy, and Management of Complications and surgical management, if warranted, is the current management.

Empiric management of suspected sepsis/BSI should include gram-positive coverage, in the presence of intravascular catheters, and broad gram-negative coverage. The choice of the antibiotic is dependent on the local epidemiology and previous microbiological data. Empiric Candida or antifungal coverage is not required, because the early initiation of antifungal has not been shown to improve the outcome in randomized controlled trials of mostly immunocompetent patients. ${ }^{16,17}$

\section{RESPIRATORY TRACT INFECTIONS}

Bacterial pneumonia is the most common cause of lower respiratory tract infections. ${ }^{18-24}$ According to Giannella and colleagues, ${ }^{25}$ community-acquired pneumonia (CAP) was found in $40.7 \%$ and hospital-acquired pneumonia (HAP) was found in $59.3 \%$ of SOT recipients treated for pneumonia. In lung transplant recipients, bacterial pneumonia or bronchitis accounts for $32 \%$ to $63 \%$ of all infections with the incidence of bacterial pneumonia peaking in the first 4 to 8 postoperative weeks and then declines by the fourth month. Perioperative antibiotics, which are focused on preoperative cultures from the recipient and donor, reduce the incidence of early bacterial pneumonia to less than $10 \% .^{20,21}$ Regarding cardiac, hepatic, and renal transplants, the incidence of early bacterial pneumonia is $15 \%, 9 \%$, and $4 \%$ to $6 \%$, respectively, ${ }^{18-20,26,27}$ with a mortality of $21 \%$ to $35 \%$ in liver and kidney transplant recipients. However, mortality between nosocomial and community-acquired infection was extreme at $58 \%$ compared with $8 \%{ }^{20-22}$ with mechanical ventilation and nosocomial infections at a higher increased risk for death. ${ }^{18,20-22,24}$ In the initial perioperative 
period, nosocomial pathogens, such as Pseudomonas aeruginosa, Escherichia coli, Klebsiella species, Acinetobacter species, and $S$ aureus, including methicillinresistant $S$ aureus (MRSA), should be considered in the immediate perioperative period; however, prolonged mechanical ventilation following transplant increases the risk for nosocomial pneumonia. ${ }^{20-22}$ Community-acquired pathogens, such as Haemophilus influenzae, Streptococcus pneumoniae, and Legionella species, may be seen. With the implementation of trimethoprim-sulfamethoxazole prophylaxis, the incidence of Nocardia pneumonia has decreased; however, it is still reported. ${ }^{20,28}$ Empiric treatment should take into consideration previous microbiological data, local epidemiology, and recent clinical history with regards to empiric antibiotic coverage.

Respiratory viral infections are a significant cause of mortality and morbidity among transplant recipients, including influenza, respiratory syncytial virus, parainfluenza virus, rhinovirus, human metapneumovirus, and coronavirus. The seasonal pattern usually follows that of the general public. ${ }^{29-31}$ Disease can consist of mild congestion and rhinorrhea to more severe tracheobronchitis, bronchiolitis, and pneumonia. Clinical manifestation can range from mild or atypical symptoms, including absence of fever, with lung transplant recipients presenting with a more severe clinical course and complications. ${ }^{29,32}$ Viral shedding is usually prolonged and seen even with the use of antivirals. ${ }^{29,32}$ Transplant recipients are at higher risk of infectious complications, including fungal and bacterial pneumonia. Respiratory viral infections appear to be a risk factor for both acute and chronic rejection, especially in lung transplant recipients. ${ }^{29,33-36}$ Diagnostic workup should consist of a nasopharyngeal swab, wash, or aspirate. If upper tract samples fail to document the cause or if there is clinical or radiological evidence of lower tract involvement, bronchoalveolar lavage (BAL) should be considered. Polymerase chain reaction (PCR)-based assays are commercially available with many centers adopting them because they are the most preferred mode of testing given the high sensitivity along with most allowing for simultaneously detecting a broad range of respiratory pathogens from a single sample. Treatment, as is outlined in Fig. 2, includes supportive care and reduction in immunosuppression.

Adenovirus is a nonenveloped, lytic double-stranded DNA virus that can be acquired de novo, through reactivation of a latent infection of the recipient, or from transplant organ. Transmission occurs by respiratory route, person-to-person contact, or fecal-oral route. The true incidence among SOT recipients is unknown, with most infections occurring within the first year after transplantation. ${ }^{37}$ Clinical manifestations can vary; however, when affecting lung transplant recipients, it can produce a range of clinical manifestations, including acute flulike illness, diffuse alveolar damage, or
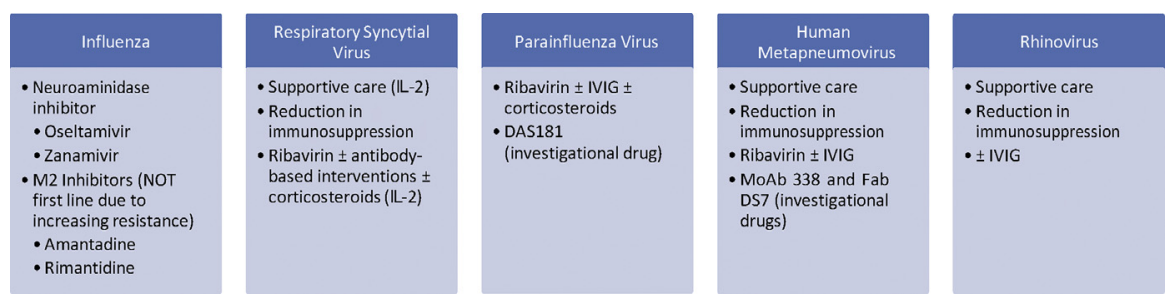

Fig. 2. Treatment recommendations for RNA respiratory viruses in SOT recipients. IL-2, interleukin-2; IVIG, intravenous immunoglobulin; MoAb, monoclonal antibody. (Data from Manuel O, Estabrook M. RNA respiratory viruses in solid organ transplantation. Am J Transplant 2013;13(s4):212-9; and Abbas S, Raybould JE, Sastry S, et al. Respiratory viruses in transplant recipients: more than just a cold. Clinical syndromes and infection prevention principles. Int J Infect Dis 2017;62:86-93.) 
necrotizing pneumonia along with chronic changes, such as bronchiolitis obliterans, interstitial fibrosis, or bronchiectasis. ${ }^{38-41}$ Viral culture, direct antigen detection, molecular methods, and histopathology are available for diagnosis with histopathologic evaluation, as the gold standard for the diagnosis of invasive disease. Rapid antigen detection kits, in particular, immunofluorescence assays when processing respiratory specimens, are commercially available, which yield rapid and specific results. PCR, qualitative and quantitative, has emerged as a widely used tool for detection because it is highly sensitive and rapid. Recovery of adenovirus from respiratory samples does not necessarily confirm disease because patients can shed asymptomatically for a prolonged period of time; therefore, it is essential to correlate with clinical findings along with detection of virus from other sites and histopathological findings. Cidofovir has the best evidence to support its use in the treatment of adenoviral infections. Brincidofovir, the lipid conjugate of cidofovir, has also demonstrated in vitro susceptibility and appears to be promising in vivo with regards to SOT recipients; however, further studies are warranted. ${ }^{42}$

CMV is a major pathogen in SOT recipients, with the ability to cause end-organ disease. The immunomodulatory effects of CMV, impaired T-cell and phagocytic function, and cytokine dysregulation can lead to opportunistic infections, rejection, graft loss, and reduced survival. ${ }^{43,44}$ The transplant recipients who are at highest risk are seronegative recipients of seropositive organ, $D+/ R-$, because they have no preexisting immunity, and seropositive recipients, $D-/ R+$, are at intermediate risk. There is little difference between $D+/ R+$ and $D-/ R-$ groups, with potentially worse outcomes in $\mathrm{D}+/ \mathrm{R}+$ (Fig. 3). ${ }^{43}$ Clinical presentation consists of dyspnea, fever, and malaise with the identification of characteristic CMV cells in lung tissue. Radiographic changes are nonspecific and include diffuse haziness, focal haziness, focal lobar consolidation, and no change. ${ }^{20}$ Diagnosis is made via cell culture viral isolation; however, this can be time consuming. Therefore, the detection of CMV DNA by PCR in the peripheral blood leukocytes, providing a sensitivity of $92 \%$ and specificity of $76 \%$ for CMV pneumonitis, along with the BAL CMV DNA PCR, is an alternative means to diagnosis. ${ }^{20,45}$ However, with regards to the BAL findings, it would be imperative to differentiate between infections versus shedding; hence, the concomitant peripheral blood leukocyte PCR along with the clinical picture is necessary to help determine the diagnosis. Treatment consists of ganciclovir or the oral alternative, valganciclovir. Foscarnet and cidofovir are alternative options; however, they are primarily reserved when there is a concern for resistance or documented resistance because their side-effect
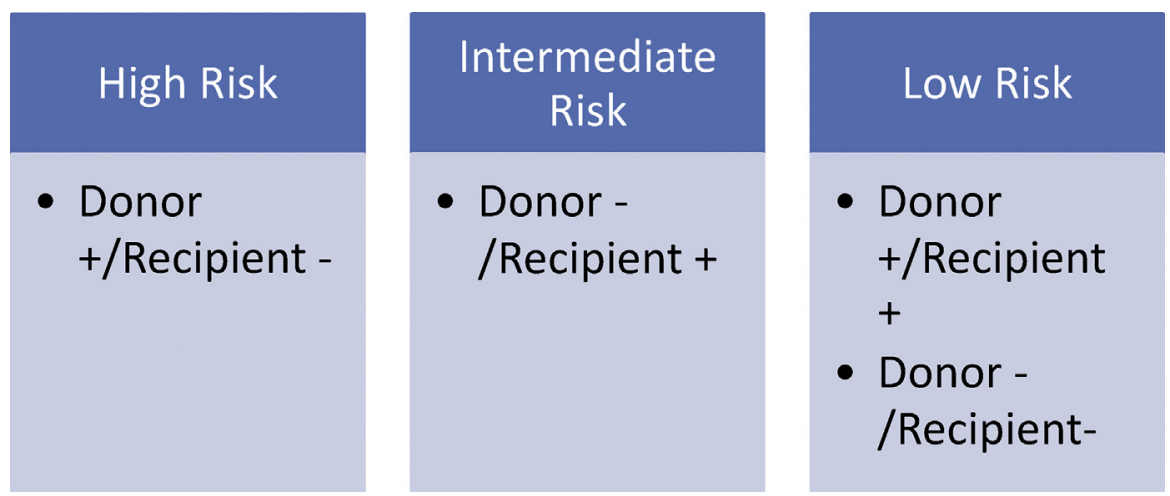

Fig. 3. CMV serostatus and the risk of complications. 
profiles are less desirable. ${ }^{20} \mathrm{CMV}$ immunoglobulin in conjunction with treatment offers limited efficacy. ${ }^{43}$ Maribavir, brincidofovir, and letermovir are novel agents that may provide alternative options for treatment; however, further studies are warranted.

Candida is a frequent colonizer of lung transplant recipients, but less than $10 \%$ of patients colonized develop invasive disease. ${ }^{20,46}$ Bronchial anastomotic infections may occur early in the postoperative setting, which can lead to anastomotic failure, parenchymal lung infection, and mediastinitis. ${ }^{47-52}$ Artificial bronchial stents can serve as a potential site for infection. ${ }^{47,48}$ Candida tracheobronchitis is based on visual inspection and histologic confirmation along with positive cultures from an appropriate specimen. ${ }^{47}$

Aspergillus species, a saprophytic organism, has higher rates of mortality, up to $54 \%$, and an incidence of $3 \%$ in lung, $2.4 \%$ in heart, $2 \%$ in liver, and $0.2 \%$ in kidney transplant recipients. ${ }^{47}$ Infection may be due to reactivation or de novo infection following inhalation of the mold. Renal failure, hemodialysis, repeated bacterial infections, leukopenia, CMV disease, high levels of immunosuppression, retransplantation, chronic exposure of the transplanted lung to the environment, and abnormal anatomic and physiologic function of the transplanted and, if still present, the native lung, airway ischemia, hypogammaglobulinemia, cystic fibrosis, and bronchial stent are all risk factors for invasive aspergillosis. ${ }^{47,53-56}$ In lung transplant recipients, $20 \%$ to $40 \%$ are colonized with Aspergillus with complicated infections affecting up to $13 \%$ of all patients. ${ }^{20,57-60}$ Colonization can also lead to bronchiolitis obliterans syndrome after lung transplantation. ${ }^{47,61}$ Clinical manifestations can range from asymptomatic colonization to tracheobronchitis, invasive pulmonary aspergillosis, empyema, and disseminated disease, ${ }^{47,62}$ with symptoms including purulent sputum, fever, malaise, respiratory distress, and hemoptysis. ${ }^{20,63}$ Aspergillus tracheobronchitis can cause airway obstruction, ulcerations, and pseudomembrane formation. ${ }^{47}$

Nonaspergillus mycelial fungi are also increasing with frequency, as high as $30 \%$, and an overall mortality of $55 \%{ }^{20,64}$; however, zygomycosis (rhizopus and mucor species) and non-aspergillus hyalohyphomycosis (scedosporium apiospermum and fusarium species) have an even higher mortality of up to $100 \% .^{20}$ Endemic fungi, Coccidioides immitis, Histoplasma capsulatum, Blastomyces dermatitidis, and cryptcoccus are additional pathogens that may need to be considered.

Radiological findings may demonstrate nodules, cavitary lesions, focal consolidation or patchy densities, wedge-shaped pleural-based lesions, air-filled bronchi with an intraluminal lesion, "air crescent" sign, and halo of decreased density. Tissue invasion by fungal organisms is the gold standard for diagnosis of invasive fungal pneumonia. However, this may be difficult to obtain; therefore, International Society for Heart and Lung Transplantation developed guidelines, A 2010 Working Formulation for the Standardization of Definitions of Infections in Cardiothoracic Transplant Recipients, to assist in the diagnosis of not only fungal infections but also bacterial and viral infections. These definitions may also be applied to abdominal transplant recipients. Treatment is as follows in Fig. 4; however, be aware of drug-drug interactions with regards to immunosuppressive agents. It is important to note, however, that empiric management of mold infections in SOT recipients is seldom necessary. The optimal approach is to pursue the diagnostic workup aggressively and treat accordingly even in lung transplant recipients.

Pneumocystis jirovecii, $T$ gondii, Mycobacterium tuberculosis, and nontuberculosis mycobacteria are other pathogens to also consider as possible causes of respiratory tract infections along with the other pathogens listed in Fig. 5. 


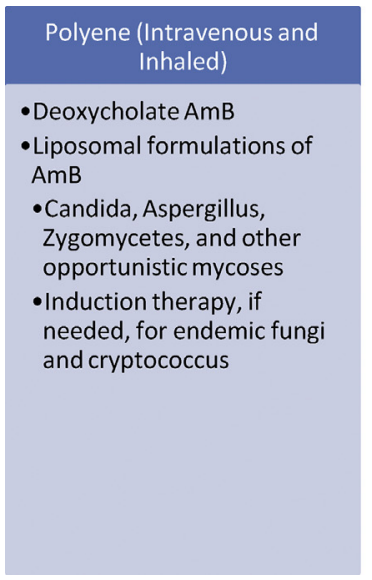

Triazoles (Intravenous and
Oral)
- Fluconazole
- C. albicans, C. tropicalis, C.
glabrata (may be dose
dependent), Coccidiodes
immitis, Cryptococcus
neoformans
- Itraconazole
- Aspergillus
- Blastomyces dermatidis
- Histoplasma capsulatum
- Voriconazole
- Aspergillus, drug of choice
- Posaconazole
- Aspergillus, Zygomycetes

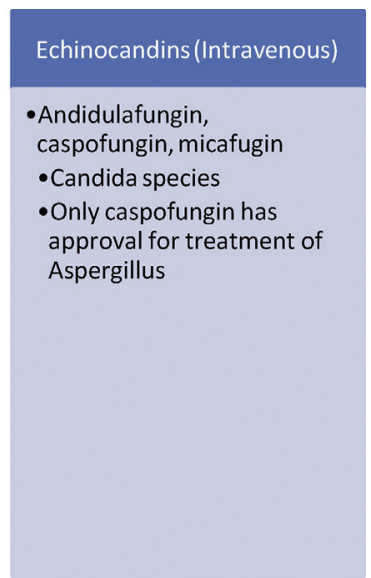

Fig. 4. Treatment of invasive fungal infections in SOT recipients.

\section{NEUROLOGIC INFECTIONS}

Central nervous system (CNS) infections can account for approximately $5 \%$ to $10 \%$ of CNS lesions in transplant recipients. ${ }^{20,64}$ Routine prophylaxis aimed at opportunistic infections along with a more conservative approach regarding immunosuppression has led to noticeable trends in infections in transplant recipients. For example, routine administration of trimethoprim-sulfamethoxazole for $P$ jirovecii has likely contributed to the reduction in infections owing to $T$ gondii, Nocardia, and Listeria monocytogenes along with acyclovir and valganciclovir attributing to the likely decline in herpesviridaerelated infections. ${ }^{65,66}$

Clinical presentation will vary and may include fever, headache, meningismus, Kernig and Brudzinski signs, new-onset seizure, papilledema, altered sensorium, and/or focal neurologic deficits; however, because of their underlying immunosuppression, these may be subtle or absent. ${ }^{65,67}$ As listed in Fig. 6, possible causes range from

Bacteria
-Community
acquired pathogens,
ie, S.pneumonia,
H.influenza,
M.cattarhalis,
Legionella
-Hospital acquired
pathogens, ie,
P.aeruguinosa and
other Gram
negative organisms,
S.aureus including
MRSA
- Nocardia
-Myocobacterium,
including
M.tuberculosis
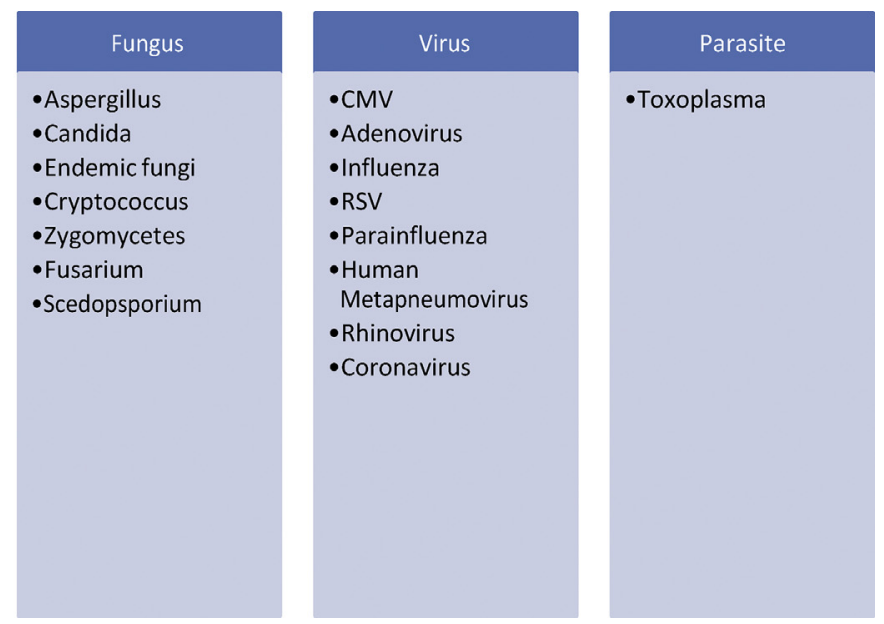

Fig. 5. Infectious causes of pulmonary complications in SOT recipients. RSV, respiratory syncytial virus. 


\begin{tabular}{l} 
Diffuse (Meningitis, Meningoencephalitis, \\
Encephalitis) \\
- Viral \\
- Herpesviridae Family (HSV, VZV, EBV, \\
CMV, HHV6) \\
- Polyomavirus \\
- West Nile Virus \\
- LCMV \\
- Rabies \\
- Enterovirus \\
- Parvovirus B19 \\
- Parasite \\
- Trypanosoma cruzi \\
- Balamuthia \\
- Naegleria \\
- Babesia microti \\
- Malaria species \\
- Fungal \\
- Endemic fungi \\
- Cryptococcus \\
- Bacterial \\
- S.pneumoniae, N.meningitidis, \\
- H.monocytogenes \\
- Hyperinfection Syndrome \\
- Treponemalos pallidum \\
- Borrelia burgdorferi \\
\hline
\end{tabular}
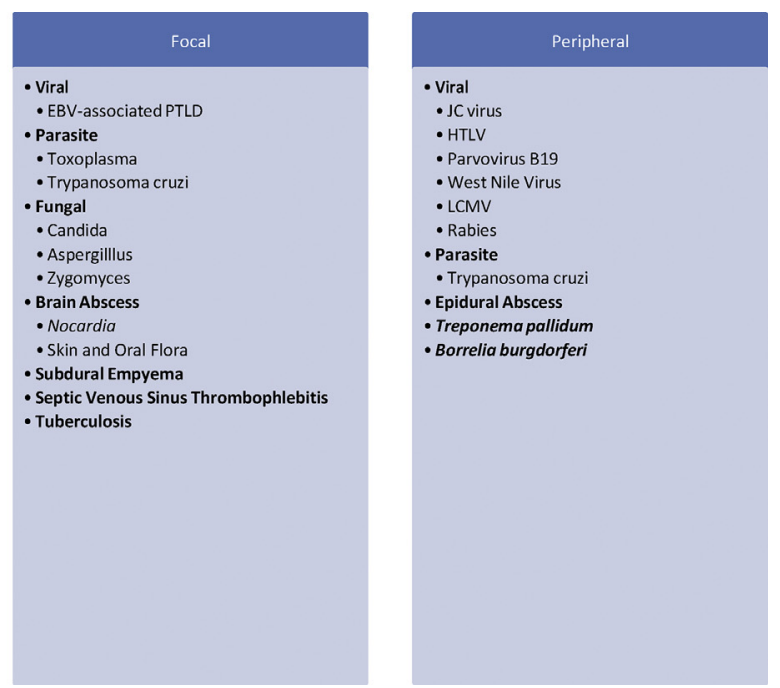

Fig. 6. Infectious causes of CNS and peripheral nervous system infections in SOT recipients. EBV, Epstein-Barr virus; LCMV, lymphocytic choriomeningitis virus; PTLD, posttransplant lymphoproliferative disease.

community-acquired organisms, donor-derived infections, reactivation, to opportunistic pathogens. Metastatic or direct lesions along with "pulmonary-brain" syndrome are also considerations in such organisms as Cryptococcus, Nocardia, Aspergillus, Zygomycetes, Strongyloides, and Toxoplasma; therefore, further investigations are warranted (eg, computed tomography of sinus and computed tomography of chest). ${ }^{2,67}$

Evaluation should include neuroimaging along with lumbar puncture as soon as possible if no contraindication exists. Cerebral spinal fluid examination should always include cell counts and differential, glucose, protein; routine smears; and cultures for bacteria, fungi, and mycobacteria. ${ }^{65,67}$ Additional specialized testing, such as viral PCR, antigen or antibody, and $16 \mathrm{~S}$ ribosomal RNA, may be required depending on the clinical scenario. ${ }^{65,67}$ However, brain biopsy with appropriate staining may be the definitive diagnosis if findings are inconclusive. Empiric treatment should cover common bacterial and viral pathogens; however, additional agents may be needed depending on the epidemiologic history.

\section{HEPATOBILIARY INFECTIONS}

Cholangitis is a common infection after liver transplant, and in fact, is the most common infection more than 1 year after liver transplant. ${ }^{68}$ Most cases occurred within 5 years and were associated with primary sclerosing cholangitis and Rouxen-Y anastomosis. ${ }^{68}$ The most frequently identified bacteria are Enterococcus spp and $E$ coli ${ }^{68,69}$; however, other gram-negative bacilli and anaerobes should also be considered. ${ }^{68,69}$

Bile leaks can occur in $2 \%$ to $25 \%$ of cases after liver transplantation, especially in living liver donor transplants. ${ }^{70}$ Clinical presentation varies with extent of the leak; however, symptoms can include abdominal pain, fever, or any sign of peritonitis. However, because of underlying immunosuppression, they can also be asymptomatic. ${ }^{70}$ In these cases, elevations in serum bilirubin, fluctuations in cyclosporine, or bilious 
ascites should raise suspicion for a bile leak. ${ }^{70}$ Biliary strictures at the site of anastomosis can also present with fever, abdominal pain, but also jaundice and asymptomatic biochemical cholestasis. ${ }^{70}$ Bilomas can represent an additional source of infection. ${ }^{41,70}$

Hepatic artery thrombosis, although more common in living donor liver transplants (LDLTs), is uncommon with deceased donor liver transplant (DDLT) with an overall incidence up to $9 \%$ and can lead to complications, including hepatic abscesses, necrosis, sepsis, and graft loss. ${ }^{71}$ Vancomycin-resistant Enterococcus faecium (VREF) is of particular concern in liver transplant. Pretransplant colonization increases rates of intra-abdominal and BSIs after transplantation. ${ }^{72,73}$ In fact, hospital and intensive care unit stays are longer for patients with VREF versus vancomycin-sensitive $E$ faecium infections. ${ }^{72}$ Liver, pancreas, and intestinal recipients are at particular risk for fungal infections, most often caused by Candida species. ${ }^{41,74,75}$

Regarding DDLT versus LDLT, there are variations with infectious complications. The rate of infection appears to be similar to DDLT; however, because of the more complex nature of the surgery, there are observable difference and specific concerns as detailed in Box 1. ${ }^{76-78}$

The clinical syndrome of hepatic dysfunction can range from mild elevated liver enzymes to hepatitis to fulminant hepatic failure. Hepatic dysfunction can present in any SOT recipient; however, of utmost concern would be liver transplant recipients. Causes can range from infectious to noninfectious with noninfectious causes primarily an issue with liver transplant recipients regarding postoperative complications, recurrence of primary disease, drug-induced complications, and rejection. ${ }^{79}$ The infectious causes listed in Fig. 7 can range from donor-derived infections, postoperative complications, community-acquired organisms, reactivation, to opportunistic pathogens. Evaluation should include imaging (eg, ultrasound, computed tomography, or MRI) along with the appropriate infectious workup (eg, blood cultures, serum PCR, serology, antigen, and/or antibodies). If the diagnosis remains inconclusive, a liver biopsy may need to be pursued with appropriate staining obtained.

\section{ENTEROCOLITIS}

Diarrhea following transplantation is frequently observed and is estimated to occur in $22 \%$ to $52 \%$ of patients. ${ }^{80-84}$ It can be associated with allograft loss and increased

\section{Box 1}

Observable differences and specific concerns regarding living donor liver transplant

- Higher incidence of biliary strictures

- Roux-en-Y choledochojejunostomy

- Increased risk of blood product transfusions

- Biliary leakage leading to bilomas

- Prolonged cholestasis and coagulopathy known as small-for-size liver syndrome

- BSI related to intra-abdominal sources (vs catheter related in DDLT)

- Contamination in abdominal cavity

- Poor premorbid host condition

Data from Abad CL, Lahr BD, Razonable RR. Epidemiology and risk factors for infection after living donor liver transplantation. Liver Transpl 2017;23(4):465-77. 


Viral
- Hepatitis family
- Herpesviridea
family
-Adenovirus
-Parvovirus B19
- LCMV
-HIV
-WNV
- Yellow Fever
-Dengue Fever
- Measles
- Rubella
-BK virus

\begin{tabular}{l}
\multicolumn{2}{c}{ Bacterial } \\
-TB \\
- Listeria \\
- Clostridum \\
difficile \\
-Routine bacteria \\
including MDRO \\
(MRSA, ESBL, CRE, \\
VRE, \\
Pseudomonas) \\
-Spirochettes \\
-Brucella \\
-Coxiella \\
-Salmonella \\
enterica serotype \\
typhi
\end{tabular}
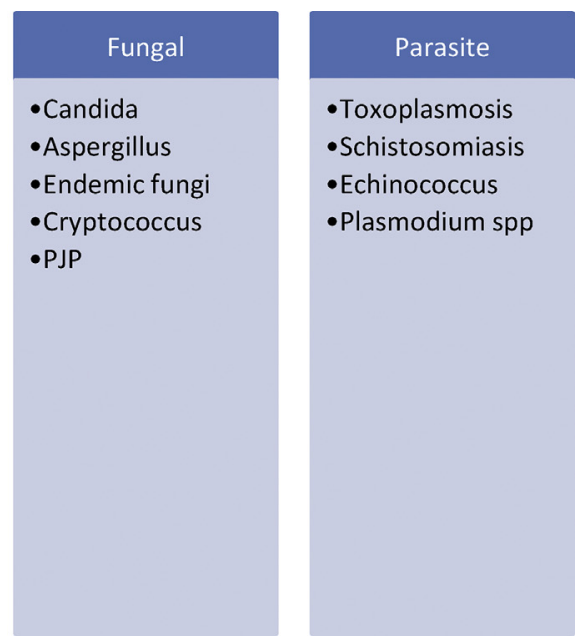

Fig. 7. Infectious causes of hepatitis in SOT recipients. HIV, human immunodeficiency virus; PJP, pneumocystis jirovecii pneumonia; TB, tuberculosis; WNV, West Nile virus. (Data from Fedoravicius A, Charlton M. Abnormal liver tests after liver transplantation. Clin Liver Dis 2016;7(4):73-9; and Talwani R, Gilliam BL, Howell C. Infectious diseases and the liver. Clin Liver Dis 2011;15(1):111-30.)

mortality. ${ }^{80,82,85,86}$ In fact, it results in 900,000 hospitalizations and 6000 deaths annually. ${ }^{87,88}$ The severity and cause of diarrhea can lead to hypovolemia and/or septic shock. Diarrhea is a recognized side effect of some immunosuppressive agents; however, infectious causes should be considered based on the clinical picture. Causes are detailed in Fig. 8. Evaluation should include stool culture, ova and parasite and giardia antigen along with appropriate PCR, antigen testing, and/or special staining. Imaging may include a computed tomography to evaluate for bowel wall edema along with

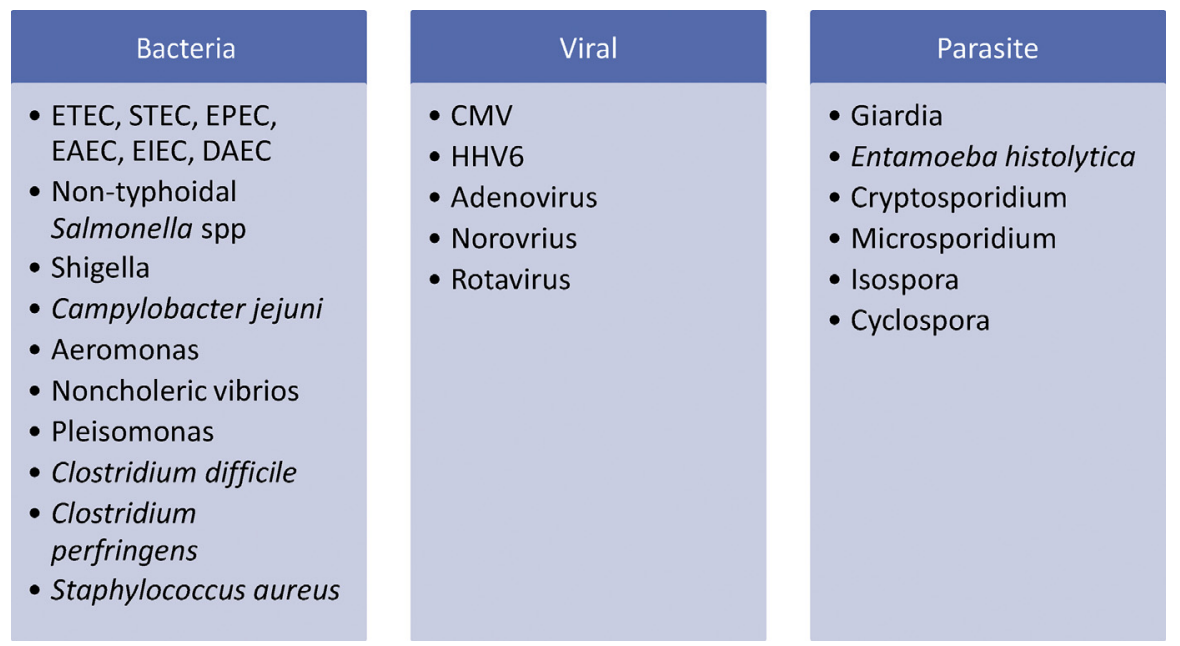

Fig. 8. Infectious causes of diarrhea in SOT recipients. DAEC, Diffusely adherent Escherichia coli; EAEC, Enteroaggregative Escherichia coli; EIEC, Enteroinvasive Escherichia coli; EPEC, Enteropathogenic Escherichia coli; ETEC, Enterotoxigenic Escherichia coli; HHV6, Human Herpesvirus 6; STEC, Shiga toxin-producing E. coli. 
colonic wall thickening and dilation. ${ }^{87}$ Esophagogastroduodenoscopy (EGD) and/or colonoscopy along with biopsy may also be warranted. CMV colitis is diagnosed via histopathology obtained during a biopsy. Serum PCR can be low to undetectable in this setting. If there is a clinical suspicion (eg, elevated serum PCR along with diarrhea, however the patient is unable to undergo colonoscopy), this may warrant empiric treatment.

Clostridium difficile infection (CDI) is among the most common health careassociated pathogen and is the most common cause of nosocomial infectious diarrhea. ${ }^{89}$ The highest incidence of CDI in SOT occurs within the first 3 months following the transplant and is likely related to frequent exposure to antimicrobials, health care settings, and immunosuppressants. ${ }^{89}$ Proton pump inhibitors (PPIs) are known to be a risk for $\mathrm{CDI}$ and may still be used in the setting of mechanical ventilation, versus $\mathrm{H} 2$ blockers. ${ }^{90}$ Hospitalized patients who use PPIs are twice as likely to develop CDI. ${ }^{89,91}$ Fulminant colitis develops in up to $13 \%$ of SOT recipients with CDI. ${ }^{89,92} \mathrm{Re}-$ lapsing disease is common, and protracted courses of therapy are often essential. ${ }^{74,93,94}$ IDSA and Society for Healthcare Epidemiology of America recently updated the Clinical Practice Guidelines for Clostridium difficile Infection in Adults and Children for 2017, and although this is for immunocompetent patients, these guidelines can be applied to SOT recipients because there have been limited studies into the treatment of CDI in SOT patients.

\section{GENITOURINARY INFECTIONS}

The most common infectious complication in SOT is UTI, accounting for $45 \%$ to $72 \%$ of all infections, and $30 \%$ of all hospitalizations for sepsis in KTRs. ${ }^{19,95-99}$ Most UTI are seen within the first 6 months after transplant; however, it can occur any time after transplantation. ${ }^{96,100,101}$ Empirical treatment is imperative, as it has been demonstrated that inappropriate antibiotic therapy is associated with an increase in mortality. ${ }^{100,102,103}$ To guide empirical therapy, local epidemiologic data, patient's microbiological history, and prior antibiotic use need to be taken into account. ${ }^{95,100}$ The most frequent organisms causing UTIs are gram-negative bacilli ${ }^{100,104}$; however, when a urinary catheter is involved, Enterococci and $S$ aureus should also be considered. The duration of treatment varies from 7-21 days depending on the clinical syndrome. Recurrent UTI, defined as 3 or more episodes of symptomatic UTIs over a 12month period or 2 in the previous 6 months, should prompt further investigation regarding anatomic and functional abnormalities along with behavioral modifications (eg, postcoital voiding) and may need a prolonged course of antibiotics, possibly 4 to 6 weeks. ${ }^{95,100,101}$ Asymptomatic bacteriuria should only be treated during the early postoperative period and up to 1 month after transplant in renal transplant patients. ${ }^{100}$

The data on UTI related to Candida spp in SOT are limited and mostly include KTR. In KTR, Candida spp are the most frequently isolated fungal cause of UTI. Unfortunately, there are no clinical trials in the management of Candida UTI in SOT. ${ }^{100}$ Candiduria is frequent, occurring in up to $11 \%$ of KTR; however, it is mostly asymptomatic. ${ }^{95}$ Asymptomatic candiduria is usually treated as there is concern regarding the allograft and potential for complications regarding the upper urinary tract; however, it should only be treated if the patient is neutropenic, or undergoing a urologic procedure. ${ }^{95,100}$ Candiduria should be classified based on risk factors for disseminated candidiasis. Urinary catheters should be removed or exchanged, and candiduria should be confirmed with a second, clean voided urine culture. Disseminated candidiasis may be considered if clinical manifestations are consistent (eg, positive blood cultures, a second urine culture after removal or replacement of the urinary catheter, funduscopic examination, cultures from any other significant site, and kidney imaging). Persistent candiduria along with 
Box 2

Duration of antibiotic therapy for urinary tract infections

- Fluconazole is the treatment of choice for cystitis and pyelonephritis

- Alternative options:

- Single dose of parenteral AMB deoxycholate with or without 5-flucytosine for cystitis; d-AMB can also be used for pyelonephritis

- AMB deoxycholate bladder irrigation for cystitis

- Liposomal AMB with or without 5-flucytosine for pyelonephritis (low concentrations in urine therefore relapse may occur)

- Echinocandins, drug of choice in unstable patients with systemic candidiasis for pyelonephritis (low concentrations in urinary tract and therefore relapse may occur)

Abbreviations: AMB, Amphotericin B; d-AMB, Amphotericin B deoxycholate.

Data from Vidal E, Cervera C, Cordero E, et al. Management of urinary tract infection in solid organ transplant recipients: consensus statement of the Group for the Study of Infection in Transplant Recipients (GESITRA) of the Spanish Society of Infectious Diseases and Clinical Microbiology (SEIMC) and the Spanish Network for Research in Infectious Diseases (REIPI). Enferm Infecc Microbiol Clin 2015;33(10):679.e1-21.

no indwelling catheters should prompt imaging of the kidneys and collecting system to exclude renal abscess, fungus balls, or other urologic abnormalities. Treatment options can range from fluconazole treatment of choice, to echinocandins, amphotericin $\mathrm{B}$, and flucytosine depending on the clinical situation and organism sensitivities with durations from at least 14 days for UTI to 2 to 4 weeks for pyelonephritis (Box 2). ${ }^{100}$

\section{SUMMARY}

SOT recipients are a complex group of patients with diverse causes given the underlying immunosuppression. As with all infectious processes, rapid identification of the

\section{Box 3}

Summary of the considerations in the management of infections in solid organ transplant recipients

\section{Bloodstream infections/sepsis}

- Always consider previous microbiological data and local epidemiology with regards to empiric antibiotics

- If an intravascular catheter is involved, consider broad gram-positive cocci, including MRSA, coverage in addition to broad gram-negative coverage, including ESBLs and CREs, if warranted

- Empiric antifungal is not needed, unless there is a high index of suspicion

Respiratory tract infections

- Always consider previous microbiological data and local epidemiology with regards to empiric antibiotics

- CAP should include empiric coverage for atypicals along with community-associated organisms

- HAP and VAP should include broad gram-positive coverage, especially MRSA, along with broad gram-negative coverage, including ESBLs and CREs if warranted

- Influenza is the only virus with approved treatment, oseltamavir; therefore, this should be started empirically if there is a concern

- Antifungals should not be started empirically, even in lung transplant recipients; however, fungal infections should be worked up thoroughly 
CNS infections

- Empiric therapy should consist of ceftriaxone and vancomycin \pm acyclovir

Hepatobiliary and gastrointestinal infections

- Always consider previous microbiological data and local epidemiology with regards to empiric antibiotics

- If VRE positive, will need to consider coverage using high-dose daptomycin or linezolid along with broad gram-negative coverage, including ESBLs and CREs, if warranted

- Regarding C difficile infections, oral vancomycin should be initiated empirically, if suspected, as first-line therapy

Genitourinary infections

- Always consider previous microbiological data along with local epidemiology with regards to empiric antibiotic decisions

- Asymptomatic bacteriuria should only be treated in renal transplant patients during the first month posttransplantation

- Antimicrobials should be tailored to the causative agent, with durations that generally range from 7 to 21 days depending on the clinical context

- Fluconazole is the treatment of choice for cystitis and pyelonephritis if Candida is the causative organism

Abbreviations: CRE, carbapenem-resistant enterobacteriaceae; ESBL, extended spectrum betalactamase inhibitors; VAP, ventilator-associated pneumonia.

pathogen, source control, and adjustment of immunosuppression is the hallmark of treatment. There is a summary of the management of infections in SOT recipients included in Box 3.

\section{REFERENCES}

1. Fernández-Ruiz M, Kumar D, Humar A. Clinical immune-monitoring strategies for predicting infection risk in solid organ transplantation. Clin Transl Immunology 2014;3(2):e12.

2. Fishman JA. From the classic concepts to modern practice. Clin Microbiol Infect 2014;20(Suppl 7):4-9.

3. Berenger BM, Doucette K, Smith SW. Epidemiology and risk factors for nosocomial bloodstream infections in solid organ transplants over a 10-year period. Transpl Infect Dis 2016;18(2):183-90.

4. Moreno A, Cervera C, Gavalda J, et al. Bloodstream infections among transplant recipients: results of a nationwide surveillance in Spain. Am J Transplant 2007; 7(11):2579-86.

5. Sanroman Budino B, Vazquez Martul E, Pertega Diaz S, et al. Autopsy-determined causes of death in solid organ transplant recipients. Transplant Proc 2004;36(3):787-9.

6. Camargo LF, Marra AR, Pignatari AC, et al. Nosocomial bloodstream infections in a nationwide study: comparison between solid organ transplant patients and the general population. Transpl Infect Dis 2015;17(2):308-13.

7. Silva M Jr, Marra AR, Pereira CA, et al. Bloodstream infection after kidney transplantation: epidemiology, microbiology, associated risk factors, and outcome. Transplantation 2010;90(5):581-7. 
8. Candel FJ, Grima E, Matesanz M, et al. Bacteremia and septic shock after solidorgan transplantation. Transplant Proc 2005;37(9):4097-9.

9. Kalil AC, Syed A, Rupp ME, et al. Is bacteremic sepsis associated with higher mortality in transplant recipients than in nontransplant patients? A matched case-control propensity-adjusted study. Clin Infect Dis 2015;60(2):216-22.

10. Kalil AC, Opal SM. Sepsis in the severely immunocompromised patient. Curr Infect Dis Rep 2015;17(6):487.

11. Kalil AC, Dakroub H, Freifeld AG. Sepsis and solid organ transplantation. Curr Drug Targets 2007;8(4):533-41.

12. Hsu J, Andes DR, Knasinski V, et al. Statins are associated with improved outcomes of bloodstream infection in solid-organ transplant recipients. Eur J Clin Microbiol Infect Dis 2009;28(11):1343-51.

13. Bodro M, Sabe N, Tubau F, et al. Risk factors and outcomes of bacteremia caused by drug-resistant ESKAPE pathogens in solid-organ transplant recipients. Transplantation 2013;96(9):843-9.

14. Ruttmann E, Bonatti H, Legit C, et al. Severe endocarditis in transplant recipients-an epidemiologic study. Transpl Int 2005;18(6):690-6.

15. Paterson DL, Dominguez EA, Chang F-Y, et al. Infective endocarditis in solid organ transplant recipients. Clin Infect Dis 1998;26(3):689-94.

16. Schuster MG, Edwards JE Jr, Sobel JD, et al. Empirical fluconazole versus placebo for intensive care unit patients: a randomized trial. Ann Intern Med 2008; 149(2):83-90.

17. Timsit JF, Azoulay E, Schwebel C, et al. Empirical micafungin treatment and survival without invasive fungal infection in adults with ICU-acquired sepsis, Candida colonization, and multiple organ failure: the EMPIRICUS randomized clinical trial. JAMA 2016;316(15):1555-64.

18. Singh N, Gayowski T, Wagener M, et al. Pulmonary infections in liver transplant recipients receiving tacrolimus. Changing pattern of microbial etiologies. Transplantation 1996;61(3):396-401.

19. Alangaden GJ, Thyagarajan R, Gruber SA, et al. Infectious complications after kidney transplantation: current epidemiology and associated risk factors. Clin Transplant 2006;20(4):401-9.

20. Singh. N CKM, Garrison. G,. Pneumonia Infection In Organ Transplant Recipients. 2017. Available at: http://www.antimicrobe.org/t35.asp. Accessed April $11,2018$.

21. Bonatti H, Pruett TL, Brandacher G, et al. Pneumonia in solid organ recipients: spectrum of pathogens in 217 episodes. Transplant Proc 2009;41(1):371-4.

22. Cervera C, Agusti C, Angeles Marcos M, et al. Microbiologic features and outcome of pneumonia in transplanted patients. Diagn Microbiol Infect Dis 2006;55(1):47-54.

23. Chan KM, Allen SA. Infectious pulmonary complications in lung transplant recipients. Semin Respir Infect 2002;17(4):291-302.

24. Sileri P, Pursell KJ, Coady NT, et al. A standardized protocol for the treatment of severe pneumonia in kidney transplant recipients. Clin Transplant 2002;16(6): 450-4.

25. Giannella M, Munoz P, Alarcon JM, et al. Pneumonia in solid organ transplant recipients: a prospective multicenter study. Transpl Infect Dis 2014;16(2): 232-41.

26. Chang GC, Wu CL, Pan SH, et al. The diagnosis of pneumonia in renal transplant recipients using invasive and noninvasive procedures. Chest 2004; 125(2):541-7. 
27. Lenner R, Padilla ML, Teirstein AS, et al. Pulmonary complications in cardiac transplant recipients. Chest 2001;120(2):508-13.

28. Wiesmayr S, Stelzmueller I, Tabarelli W, et al. Nocardiosis following solid organ transplantation: a single-centre experience. Transpl Int 2005;18(9):1048-53.

29. Manuel O, Estabrook M. RNA respiratory viruses in solid organ transplantation. Am J Transplant 2013;13(s4):212-9.

30. Couch RB, Englund JA, Whimbey E. Respiratory viral infections in immunocompetent and immunocompromised persons. Am J Med 1997;102(3a):2-9 [discussion: 25-6].

31. Lopez-Medrano F, Aguado JM, Lizasoain M, et al. Clinical implications of respiratory virus infections in solid organ transplant recipients: a prospective study. Transplantation 2007;84(7):851-6.

32. Ison MG. Respiratory viral infections in transplant recipients. Antivir Ther 2007; 12(4 Pt B):627-38.

33. Billings JL, Hertz MI, Savik K, et al. Respiratory viruses and chronic rejection in lung transplant recipients. J Heart Lung Transplant 2002;21(5):559-66.

34. Khalifah AP, Hachem RR, Chakinala MM, et al. Respiratory viral infections are a distinct risk for bronchiolitis obliterans syndrome and death. Am J Respir Crit Care Med 2004;170(2):181-7.

35. Kumar D, Erdman D, Keshavjee S, et al. Clinical impact of community-acquired respiratory viruses on bronchiolitis obliterans after lung transplant. Am J Transplant 2005;5(8):2031-6.

36. Milstone A, Brumble L, Barnes J, et al. A single-season prospective study of respiratory viral infections in lung transplant recipients. Eur Respir J 2006;28(1): $131-7$.

37. Abbas S, Raybould JE, Sastry S, et al. Respiratory viruses in transplant recipients: more than just a cold. Clinical syndromes and infection prevention principles. Int J Infect Dis 2017;62:86-93.

38. Florescu DF, Hoffman JA. Adenovirus in solid organ transplantation. Am J Transplant 2013;13(s4):206-11.

39. Ohori NP, Michaels MG, Jaffe R, et al. Adenovirus pneumonia in lung transplant recipients. Hum Pathol 1995;26(10):1073-9.

40. Bridges ND, Spray TL, Collins $M H$, et al. Adenovirus infection in the lung results in graft failure after lung transplantation. J Thorac Cardiovasc Surg 1998;116(4): 617-23.

41. Humar A, Doucette K, Kumar D, et al. Assessment of adenovirus infection in adult lung transplant recipients using molecular surveillance. J Heart Lung Transplant 2006;25(12):1441-6.

42. Hirsch HH, Martino R, Ward KN, et al. Fourth European Conference on Infections in Leukaemia (ECIL-4): guidelines for diagnosis and treatment of human respiratory syncytial virus, parainfluenza virus, metapneumovirus, rhinovirus, and coronavirus. Clin Infect Dis 2013;56(2):258-66.

43. Haidar G, Singh N. Viral infections in solid organ transplant recipients: novel updates and a review of the classics. Curr Opin Infect Dis 2017;30(6):579-88.

44. Singh N. Late-onset cytomegalovirus disease as a significant complication in solid organ transplant recipients receiving antiviral prophylaxis: a call to heed the mounting evidence. Clin Infect Dis 2005;40(5):704-8.

45. Michaelides A, Liolios L, Glare EM, et al. Increased human cytomegalovirus (HCMV) DNA load in peripheral blood leukocytes after lung transplantation correlates with HCMV pneumonitis. Transplantation 2001;72(1):141-7. 
46. Flume PA, Egan TM, Paradowski LJ, et al. Infectious complications of lung transplantation. Impact of cystic fibrosis. Am J Respir Crit Care Med 1994;149(6): 1601-7.

47. Shoham S, Marr KA. Invasive fungal infections in solid organ transplant recipients. Future Microbiol 2012;7(5):639-55.

48. Kubak BM. Fungal infection in lung transplantation. Transpl Infect Dis 2002; 4(Suppl 3):24-31.

49. Wahidi MM, Willner DA, Snyder LD, et al. Diagnosis and outcome of early pleural space infection following lung transplantation. Chest 2009;135(2):484-91.

50. Schaenman JM, Rosso F, Austin JM, et al. Trends in invasive disease due to Candida species following heart and lung transplantation. Transpl Infect Dis 2009;11(2):112-21.

51. Horvath J, Dummer S, Loyd J, et al. Infection in the transplanted and native lung after single lung transplantation. Chest 1993;104(3):681-5.

52. Hadjiliadis D, Howell DN, Davis RD, et al. Anastomotic infections in lung transplant recipients. Ann Transplant 2000;5(3):13-9.

53. Gavalda J, Len O, San Juan R, et al. Risk factors for invasive aspergillosis in solid-organ transplant recipients: a case-control study. Clin Infect Dis 2005; 41(1):52-9.

54. Rosenhagen M, Feldhues R, Schmidt J, et al. A risk profile for invasive aspergillosis in liver transplant recipients. Infection 2009;37(4):313-9.

55. Singh N, Pruett TL, Houston S, et al. Invasive aspergillosis in the recipients of liver retransplantation. Liver Transpl 2006;12(8):1205-9.

56. Fortún J, Martín-Dávila P, Moreno S, et al. Risk factors for invasive aspergillosis in liver transplant recipients. Liver Transpl 2002;8(11):1065-70.

57. Iversen M, Burton CM, Vand S, et al. Aspergillus infection in lung transplant patients: incidence and prognosis. Eur J Clin Microbiol Infect Dis 2007;26(12): 879-86.

58. Kotloff RM, Ahya VN, Crawford SW. Pulmonary complications of solid organ and hematopoietic stem cell transplantation. Am J Respir Crit Care Med 2004; 170(1):22-48.

59. Mehrad B, Paciocco G, Martinez FJ, et al. Spectrum of Aspergillus infection in lung transplant recipients: case series and review of the literature. Chest 2001;119(1):169-75.

60. Monforte V, Roman A, Gavalda J, et al. Nebulized amphotericin B prophylaxis for Aspergillus infection in lung transplantation: study of risk factors. J Heart Lung Transplant 2001;20(12):1274-81.

61. Weigt SS, Elashoff RM, Huang C, et al. Aspergillus colonization of the lung allograft is a risk factor for bronchiolitis obliterans syndrome. Am J Transplant 2009; 9(8):1903-11.

62. Grossi P, Farina C, Fiocchi R, et al. Prevalence and outcome of invasive fungal infections in 1,963 thoracic organ transplant recipients: a multicenter retrospective study. Italian Study Group of Fungal Infections in Thoracic Organ Transplant Recipients. Transplantation 2000;70(1):112-6.

63. Westney GE, Kesten S, De Hoyos A, et al. Aspergillus infection in single and double lung transplant recipients. Transplantation 1996;61(6):915-9.

64. Husain S, Alexander BD, Munoz P, et al. Opportunistic mycelial fungal infections in organ transplant recipients: emerging importance of non-Aspergillus mycelial fungi. Clin Infect Dis 2003;37(2):221-9.

65. Smith JA. CNS infections in solid organ transplant recipients. Hospital Physician Neurology Board manual 2005;9(3):1-15. 
66. Singh N, Husain S. Infections of the central nervous system in transplant recipients. Transpl Infect Dis 2000;2(3):101-11.

67. Wright AJ, Fishman JA. Central nervous system syndromes in solid organ transplant recipients. Clin Infect Dis 2014;59(7):1001-11.

68. Aberg F, Makisalo H, Hockerstedt K, et al. Infectious complications more than 1 year after liver transplantation: a 3-decade nationwide experience. Am J Transplant 2011;11(2):287-95.

69. Raakow R, Bechstein WO, Kling N, et al. The importance of late infections for the long-term outcome after liver transplantation. Berlin (Germany): Springer Berlin Heidelberg; 1996. p. 155-6.

70. Kochhar G, Parungao JM, Hanouneh IA, et al. Biliary complications following liver transplantation. World J Gastroenterol 2013;19(19):2841-6.

71. Mourad MM, Liossis C, Gunson BK, et al. Etiology and management of hepatic artery thrombosis after adult liver transplantation. Liver Transpl 2014;20(6): 713-23.

72. Singh N. Infections in solid-organ transplant recipients. Am J Infect Control 1997:25(5):409-17.

73. Pessoa MG, Terrault NA, Ferrell LD, et al. Hepatitis G virus in patients with cryptogenic liver disease undergoing liver transplantation. Hepatology 1997;25(5): 1266-70.

74. O'Shea DT, Humar A. Life-threatening infection in transplant recipients. Crit Care Clin 2013;29(4):953-73.

75. Person AK, Kontoyiannis DP, Alexander BD. Fungal infections in transplant and oncology patients. Infect Dis Clin North Am 2010;24(2):439-59.

76. Abad CL, Lahr BD, Razonable RR. Epidemiology and risk factors for infection after living donor liver transplantation. Liver Transpl 2017;23(4):465-77.

77. Kim JE, Oh SH, Kim KM, et al. Infections after living donor liver transplantation in children. J Korean Med Sci 2010;25(4):527-31.

78. Kim YJ, Kim SI, Wie SH, et al. Infectious complications in living-donor liver transplant recipients: a 9-year single-center experience. Transpl Infect Dis 2008; 10(5):316-24.

79. Fedoravicius A, Charlton M. Abnormal liver tests after liver transplantation. Clin Liver Dis 2016;7(4):73-9.

80. Echenique IA, Penugonda S, Stosor V, et al. Diagnostic yields in solid organ transplant recipients admitted with diarrhea. Clin Infect Dis 2015;60(5):729-37.

81. Maes B, Hadaya K, de Moor B, et al. Severe diarrhea in renal transplant patients: results of the DIDACT study. Am J Transplant 2006;6(6):1466-72.

82. Bunnapradist S, Neri L, Wong W, et al. Incidence and risk factors for diarrhea following kidney transplantation and association with graft loss and mortality. Am J Kidney Dis 2008;51(3):478-86.

83. Herrero JI, Benlloch S, Bernardos A, et al. Gastrointestinal complications in liver transplant recipients: MITOS study. Transplant Proc 2007;39(7):2311-3.

84. Gil-Vernet S, Amado A, Ortega F, et al. Gastrointestinal complications in renal transplant recipients: MITOS study. Transplant Proc 2007;39(7):2190-3.

85. Nagaraj N, Kahan B, Adler DG. Gastrointestinal complications in renal transplant patients: a large, single-center experience. Dig Dis Sci 2007;52(12): 3394-5.

86. Altiparmak MR, Trablus S, Pamuk ON, et al. Diarrhoea following renal transplantation. Clin Transplant 2002;16(3):212-6.

87. Subramanian A. Diarrhea in organ transplant recipients. 2010. Available at: http://www.antimicrobe.org/new/t12_dw.html. Accessed April 11, 2018. 
88. Thielman NM, Guerrant RL. Clinical practice. Acute infectious diarrhea. N Engl J Med 2004;350(1):38-47.

89. Ward SD, ER. Clostridium difficile Infection in Solid Organ Transplant Patients. 2017. Available at: http://www.antimicrobe.org/t40.asp. Accessed April 2018.

90. MacLaren R, Reynolds PM, Allen RR. Histamine-2 receptor antagonists vs proton pump inhibitors on gastrointestinal tract hemorrhage and infectious complications in the intensive care unit. JAMA Intern Med 2014;174(4):564-74.

91. Dubberke ER, Gerding DN, Classen D, et al. Strategies to prevent clostridium difficile infections in acute care hospitals. Infect Control Hosp Epidemiol 2008; 29(Suppl 1):S81-92.

92. Dallal RM, Harbrecht BG, Boujoukas AJ, et al. Fulminant Clostridium difficile: an underappreciated and increasing cause of death and complications. Ann Surg 2002;235(3):363-72.

93. Riddle DJ, Dubberke ER. Clostridium difficile infection in solid organ transplant recipients. Curr Opin Organ Transplant 2008;13(6):592-600.

94. Albright JB, Bonatti H, Mendez J, et al. Early and late onset Clostridium difficileassociated colitis following liver transplantation. Transpl Int 2007;20(10):856-66.

95. Parasuraman R, Julian K. Urinary tract infections in solid organ transplantation. Am J Transplant 2013;13(s4):327-36.

96. Alangaden $\mathrm{G}$. Urinary tract infections in renal transplant recipients. Curr Infect Dis Rep 2007;9(6):475-9.

97. Abbott KC, Oliver JD 3rd, Hypolite I, et al. Hospitalizations for bacterial septicemia after renal transplantation in the United States. Am J Nephrol 2001; 21(2):120-7.

98. Veroux M, Giuffrida G, Corona D, et al. Infective complications in renal allograft recipients: epidemiology and outcome. Transplant Proc 2008;40(6):1873-6.

99. Pelle G, Vimont S, Levy PP, et al. Acute pyelonephritis represents a risk factor impairing long-term kidney graft function. Am J Transplant 2007;7(4):899-907.

100. Vidal E, Cervera C, Cordero E, et al. Management of urinary tract infection in solid organ transplant recipients: Consensus statement of the Group for the Study of Infection in Transplant Recipients (GESITRA) of the Spanish Society of Infectious Diseases and Clinical Microbiology (SEIMC) and the Spanish Network for Research in Infectious Diseases (REIPI). Enferm Infecc Microbiol Clin 2015;33(10):679.e1-e21.

101. Munoz P. Management of urinary tract infections and lymphocele in renal transplant recipients. Clin Infect Dis 2001;33(Suppl 1):S53-7.

102. Kang Cl, Kim SH, Park WB, et al. Bloodstream infections caused by antibioticresistant gram-negative bacilli: risk factors for mortality and impact of inappropriate initial antimicrobial therapy on outcome. Antimicrob Agents Chemother 2005;49(2):760-6.

103. Schwaber MJ, Carmeli Y. Mortality and delay in effective therapy associated with extended-spectrum beta-lactamase production in Enterobacteriaceae bacteraemia: a systematic review and meta-analysis. J Antimicrob Chemother 2007; 60(5):913-20.

104. Cervera C, Linares L, Bou G, et al. Multidrug-resistant bacterial infection in solid organ transplant recipients. Enferm Infecc Microbiol Clin 2012;30(Suppl 2): 40-8. 